\title{
Unterarm - Strategien bei fehlgeschlagenen Osteosynthesen
}

Andreas Harbrecht, Michael Hackl, Kilian Wegmann, Lars Peter Müller

\section{Häufigkeit von Unterarmfrakturen und Darstellung der potenziellen Komplikationen}

\section{Grundlagen}

Der Unterarm stellt eine wichtige funktionelle Einheit zwischen dem Ellenbogen- und dem Handgelenk dar. Umwendbewegungen werden mithilfe der Rotation des Radius um die Ulna an die Hand weitergegeben. Diese Bewegung ist im alläglichen Leben von immenser Bedeutung. Die Membrana interossea antebrachii zwischen Radius und Ulna stellt zudem eine wichtige anatomische Struktur zur Stabilisierung dieser Bewegung und der gleichmäßigen Kraftübertragung bei von außen einwirkenden Kräften dar [1 -3].

Zu den Unterarmfrakturen zählen [4, 5]:

- Ulna- und Radiusschaftfraktur

- Galeazzi-Fraktur (Radiusschaftfraktur mit Luxation der distalen Ulna aus dem distalen Radioulnargelenk)

- Essex-Lopresti-Fraktur (proximale Fraktur des Radius, komplette Ruptur der Membrana interossea, Luxation des distalen Radioulnargelenks) [6]

Monteggia-Verletzungen und Monteggia-like Lesions werden aufgrund ihrer Komplexität und anatomischen Nähe häufig den Ellenbogenfrakturen zugeordnet, weshalb von einer Beschreibung in diesem Artikel abgesehen wird.

Frakturen im Bereich des Unterarmes kommen mit einer Häufigkeit von 13,8/100 000 vor [7] und Frakturen beider Röhrenknochen am Unterarm machen einen Anteil von 1-2\% aller Extremitätenfrakturen aus [8]. Ursache sind meist Unfälle bei sportlicher Aktivität oder hoher Energieeinwirkung. Demzufolge sind meist junge Patienten betroffen [9].

Unterarmfrakturen sollten, aufgrund der nicht zufriedenstellenden Ergebnisse unter konservativer Therapie, der operativen Versorgung zugeordnet werden. Ausgenommen hiervon sind sog. „Parierfrakturen“ der Ulna (im Schaftbereich, nicht disloziert), die auch unter konservativer Therapie gut ausheilen.
Das operative Mittel der Wahl ist die Plattenosteosynthese. Hierbei wird auf Kompressionsplatten bei einfachen Frakturen und auf winkelstabile Systeme bei komplexen Mehrfragmentfrakturen zurückgegriffen. Bei der Primärversorgung sind i.d.R. 3,5-mm-Platten ausreichend. Auch Marknägel können verwendet werden, dabei steht ein geringeres Weichteiltrauma bei Implantation einer geringeren Rotationsstabilität, die insbesondere am Radius wirkt, gegenüber [10]. Abschließend geklärt ist die Favorisierung eines der o.g. Osteosyntheseverfahren jedoch noch nicht. Beide Techniken zeigen aktuell keine Unterschiede in der Rate an erfolgreich verheilten Frakturen [10]. Die Plattenosteosynthese bleibt die von der AO (Arbeitsgemeinschaft für Osteosynthesefragen), vor allem in der Revisionschirurgie, empfohlene Osteosynthesetechnik.

\section{Komplikationen}

Unterarmschaftfrakturen heilen bei primärer operativer Versorgung in $98 \%$ der Fälle zufriedenstellend aus [1114]. Angaben über die Häufigkeit von Pseudarthrosen variieren in der Literatur von 2 bis $10 \%$ [11, 13, 15-17]. Pseudarthrosen sind am Unterarm meist der Gruppe der oligotrophen bzw. atrophen und nur in $9 \%$ den hypertrophen Ausprägungsformen zuzuordnen [18].

In der Literatur wird die Rate an insgesamt seltenen postoperativen Synostosen zwischen Ulna und Radius mit 0,3-3,8\% angegeben, wobei diese Form der Komplikation nicht vom Osteosyntheseimplantat abhängig ist [8]. Die Membrana interossea sollte möglichst intraoperativ geschont und die Schraubenlänge exakt gewählt werden [8]. Darüber hinaus häufen sich Fälle von Synostosen bei Patienten mit schwerem Schädel-Hirn-Trauma und/oder polytraumatisierten Patienten [19].

Malunions am Unterarm können Auswirkungen auf die Funktion der Extremität haben. So können die Griffkraft, Rotationsfähigkeit und die Range of Motion des Ellenbogens und/oder des Handgelenkes davon betroffen sein. Eine spontane Korrektur von in Fehlstellung verheilten Unterarmfrakturen ist nur im Kindesalter bis maximal 9 Jahre zu erwarten [20]. Sollte es zu Schmerzen, manifestem Rotations- und Griffkraftverlust kommen, ist eine Korrekturosteotomie als chirurgische Revisionsoption möglich. 


\section{Risikofaktoren für die Ausbildung von Pseudarthrosen}

Das Risiko für die Ausbildung einer Pseudarthrose als Hauptfaktor einer fehlgeschlagenen Osteosynthese hängt von der Frakturlokalisation, dem Verletzungsmuster, den allgemeinen Patientenfaktoren, einer etwaigen Infektion und der operativen Technik ab.

\section{Allgemeine Risikofaktoren}

Allgemeine Risikofaktoren sind Diabetes mellitus, Übergewicht und hohes Alter [21-23]. In einzelnen Studien konnte auch ein erhöhtes Risiko für Pseudarthrosen bei Rauchern festgestellt werden [24].

\section{Infektion}

Infektionspseudarthrosen stellen sich i.d.R. als atrophe Ausprägungsformen dar. Risiken sind offene Verletzungen und o.g. allgemeine Faktoren. Eine postoperative Infektion ist häufig die Ursache einer fehlgeschlagenen Osteosynthese. Genaue Daten zur Rate an Infektpseudarthrosen am Unterarm liegen aktuell noch nicht vor. Einzelne Fallstudien berichten von einem Anteil von $25 \%$, bei denen Infektionen die Ursache von Pseudarthrosen sind [25].

\section{Frakturlokalisation und Verletzungsmuster}

Frakturen im Bereich des mittleren Schaftdrittels erscheinen häufiger mit einer postoperativen Pseudarthrose vergesellschaftet [26]. Zudem ist die Wahrscheinlichkeit bei offenen Frakturen und Mehrfragmentfrakturen, die häufiger im Rahmen von Hochrasanztraumata entstehen, höher [27].

\section{Operative Technik}

Technische Fehler spielen vor allem bei der Versorgung komplexerer Frakturen eine wichtige Rolle. So konnten in mehreren Fallserien eindeutige operative Fehler identifiziert werden. Hierzu zählen [17, 19]:

- die Wahl einer zu kurzen Platte

- inkorrekte Zentrierung der Platte über der Fraktur

- Einbringung der Schrauben zu nah an der Fraktur

- Verwendung von zu wenigen Schrauben zur Verankerung der Platte

Es sollte möglichst weichteilschonend gearbeitet werden. Auch die Ablösung des Periostes sollte so gering wie möglich ausfallen, um die Blutversorgung nicht unnötigerweise zusätzlich zu kompromittieren. Der zu groß ausgefallene OP-Zugang mit ausgeprägter Verletzung der Biologie des Knochens stellt einen wesentlichen Risikofaktor für die potenziell fehlschlagende Osteosynthese dar [21]. Die Implantatwahl bei korrekt indizierter Fraktur (Kompression vs. winkelstabil) hat hingegen keine Auswirkung auf das potenzielle Versagen einer primären Osteosynthese [28].

\section{Revisionsstrategien bei fehlgeschla- genen Osteosynthesen - Techniken}

\section{Generelle Vorgehensweisen}

Die Etablierung einer passenden Vorgehensweise bei fehlgeschlagenen Osteosynthesen am Unterarm beinhaltet zwingend die genaue Kenntnis der Ursache des Scheiterns. Fragen, die zu stellen sind:

- War die primäre Stabilität ausreichend?

- Liegt ein Infekt vor?

- Ist die Frakturbiologie ausreichend berücksichtigt worden?

- Welche externen Faktoren (Patient, erneutes Trauma) liegen vor?

Die genaue Analyse der zugrunde liegenden Problematik sollte am Anfang jeder Revisionstherapie stehen. Bei externen Patienten bietet es sich an, den OP-Bericht der primären Operation einzuholen, um evtl. Rückschlüsse auf das Scheitern oder Informationen über wichtige, durch die Operation tendenziell veränderte Strukturen am Unterarm, zu erhalten. Bei Reosteosynthese sind die Bohrlöcher möglichst divergierend zu den bereits vorhandenen Bohrlöchern der primären Osteosynthese zu setzen, um eine bestmögliche Verankerung der Platte zu gewährleisten. Für den Erfolg der Revision sind neben der mechanischen Stabilität auch biologische Faktoren wie erhaltene Durchblutung, vor Ort vorhandene Wachstumsfaktoren und knochenbildende Zellen, von Bedeutung.

Der Verfahrenswechsel auf einen Marknagel ist bei Unterarmschaftpseudarthrosen denkbar [29]. Wie bereits geschildert, ist dabei die geringere Rotationsstabilität und Kompression zu berücksichtigen. Da aktuell keine vergleichenden Studien zwischen Marknagel- und Plattenosteosynthese in der Revisionschirurgie am Unterarm vorliegen und die Datenlage für Marknägel generell eher spärlich ist, ist der Einsatz dieser Technik eher als zurückhaltend zu bezeichnen.

Die Revisionschirurgie an der oberen Extremität stellt für den behandelnden Chirurgen eine große Herausforderung dar. Es stehen jedoch zahlreiche Verfahren zur Auswahl, um eine Revisionsoperation erfolgreich durchzuführen. Die Wahl eines dieser Verfahren stützt sich dabei auf die persönliche Expertise des Operateurs und seine theoretisch fundierten Überzeugungen [29].

\section{Pseudarthrosen}

Pseudarthrosen am Unterarm bestehen zu einem großen Anteil aus oligotrophen bzw. atrophen Pseudarthrosen [18]. Demnach ist häufig nicht die mechanische Stabilität das Problem, sondern eine unzureichende biologische Aktivität. Um dieses Problem erfolgreich zu sanieren, ist eine Form der Augmentation des ehemaligen Frakturspaltes notwendig. Diese Augmentation kann aus einer 

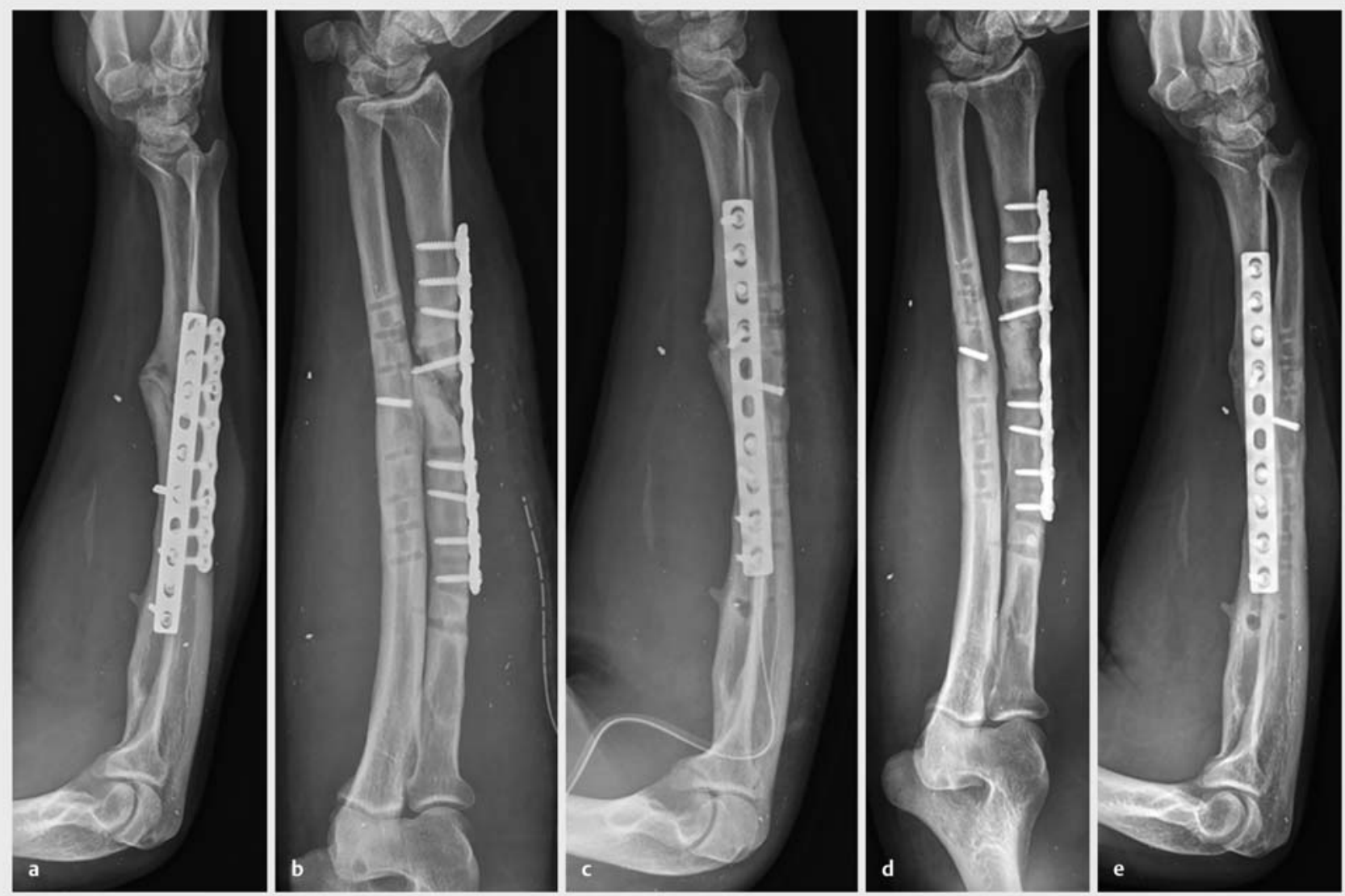

- Abb. 1 Bild einer hypertrophen Pseudarthrose nach Bombenexplosion und mehrmaligen Voroperationen im Ausland und Revisionsergebnis. a Präoperativ lateraler Strahlengang. b, c Unmittelbar postoperativ nach Pseudarthrosen-Débridement, Materialentfernung (ME) Ulna und verbliebener abgebrochener Schraube, Augmentation mittels Spongiosaplastik aus Beckenkamm und Reosteosynthese mittels LC-DCP 3,5 mm in a.-p. und lateraler Ansicht. d, e Ergebnis 3 Monate nach OP in a.-p. und lateraler Ansicht.

Spongiosaplastik, der Interposition eines vaskularisierten Knochenspans oder der Interposition eines nicht vaskularisierten Knochenspans bestehen. Die Ergebnisse in der Revisionschirurgie der Pseudarthrosen am Unterarm zeigen insgesamt ein gutes Outcome. Mithilfe der o.g. Verfahren, kombiniert mit lokalem Débridement und stabiler Reosteosynthese, können viele Pseudarthrosen erfolgreich behandelt werden ( $\bullet$ Abb. $\mathbf{1} \mathbf{a}-\mathbf{e}$ ) [30].

\section{Infektpseudarthrosen}

Infektpseudarthrosen stellen eine große Herausforderung für den behandelnden Chirurgen dar. In der Regel handelt es sich um eine atrophe Pseudarthrose, die debridiert werden muss. Eingebrachtes Osteosynthesematerial muss entfernt und Proben zur mikrobiologischen Aufarbeitung asserviert werden. Anzuschließen ist eine Sanierungsphase, bspw. durch eine systemische resistogrammgerechte Antiobiotikatherapie, lokale Antiobiotikaketten oder antibiotikabehaftete Spacer. Zwischenzeitlich kann eine frakturferne Stabilisierung mittels Fixateur externe erfolgen. Nach der Sanierungsphase ist i.d.R. eine Augmentation notwendig, die durch eine stabile Os- teosynthese fixiert werden sollte. Als Alternative können hierbei die im weiteren Text erklärte Masquelet-Technik und der Segmenttransport dienen.

\section{Spongiosaplastik}

Die alleinige Spongiosaplastik kann bei vielen Defekten angewendet werden. Zu berücksichtigen ist jedoch, dass die Stabilität bei allzu großen Defekten nicht ausreichend sein kann. Wo genau die Linie zu ziehen ist zwischen noch realisierbarer Spongiosaplastik und Augmentation mithilfe eines kortikospongiösen Spans, ist nicht eindeutig geklärt. Einzelne Studien berichten über eine Defektlänge von bis zu $6 \mathrm{~cm}$, die erfolgreich mit einer Spongiosaplastik behandelt werden könne [18]. Der Vorteil der Spongiosaplastik liegt in der geringeren Entnahmemorbidität. Autologe Knochenspäne, die i.d. R. aus dem Beckenkamm, der Fibula oder der Tibia entnommen werden, führen häufig zu Schmerzen an der Entnahmestelle und selten auch zu Deformierungen. Zusammengefasst kann eine autologe Spongiosaplastik bei kleineren Defekten empfohlen werden ( $\triangleright$ Abb. 1 a-e). Einigkeit über die Indikationsgrenze, bezogen auf die Defektlänge, besteht aktuell noch nicht. 


\section{Interposition eines nicht vaskularisierten Grafts}

Die Augmentation mit einem kortikospongiösen autologen Graft, i. d. R. aus dem Beckenkamm, der Fibula oder der Tibia, zeigt gute Ergebnisse. Mit dieser Form der Therapie kann in 63-100\% eine Ausheilung erreicht werden [31 - 34]. Als überbrückende Reosteosynthese sollte eine ausreichende Plattenlänge gewählt und die Biologie der Augmentation nicht durch unnötige Schrauben in unmittelbarer Nähe des Implantates belastet werden. In der eigenen Praxis wird der Knochenspan aus dem Beckenkamm entnommen. Sollte dieser nicht ausreichen, kann die Augmentation durch zusätzliche Spongiosa, aus dem i. d.R. ipsilateralen Femur, die mithilfe eines Reamer-Irrigator-Aspirator (RIA ${ }^{\circledR}$, Fa. Synthes) gewonnen wird, unterstützt werden [35]. Als weitere Option, sollte eine Beckenkammentnahme nicht (mehr) möglich sein, dient ein Fibulagraft.

Analog zu der Spongiosaplastik stellt sich auch hier die Frage nach der maximalen Länge, die ein Defekt haben darf, um noch erfolgreich mittels eines autologen Grafts behandelt werden zu können. Es bleibt letztlich unklar, ob auch hier eine Defektlänge von $6 \mathrm{~cm}$ als Grenze anzusehen ist [36].

\section{Interposition eines vaskularisierten Grafts}

Der am häufigsten verwendete Graft bei der Interposition mit einem vaskularisierten Knocheninterponat ist die Fibula $[37,38]$. Es existieren auch einige Fallserien, in denen ein lokales Autograft von der distalen Ulna oder dem Radius mit Anschluss an die A. interosseus posterior verwendet wurde [39]. Die Verwendung eines vaskularisierten Autografts erzielt in den wenigen vorhandenen Fallstudien gute Ergebnisse von 75-100\% Ausheilungsraten [40-42]. Verbunden ist diese operative Technik mit einem großen Hebungsschaden des Grafts, Mikrochirurgie, längerer Operationsdauer sowie zahlreichen Komplikationen. Häufig erfordert diese Art der Operation die Kooperation mit den Kollegen der Plastischen Chirurgie.

Zusammengefasst kann die Interposition mit einem autologen vaskularisierten Graft nicht als Primäroption in der Revisionschirurgie empfohlen werden und dient als profunde Ausweichoption, falls die Implantation eines autologen nicht vaskularisierten Knocheninterponats fehlschlägt oder der Defekt so groß ist, dass eine kombinierte Deckung mit Knochen, Muskel und Haut notwendig wird.

\section{Autologer Graft vs. allogener Graft}

Die biologische Aktivität eines autologen Grafts ist der eines allogenen Grafts deutlich überlegen und sollte daher die Primäroption darstellen [43]. Im eigenen Vorgehen wird auf allogenes Material zurückgegriffen, sollten alle autologen Optionen (Knochenspäne, Addition von Spongiosa) ausgeschöpft sein und das allogene Material rein additiv zur Anwendung kommen.

\section{Masquelet-Technik}

Grundlage dieses Verfahrens ist die sog. „Membraninduktion“. Diese beruht darauf, dass nach der Resektion einer i.d.R. Infektpseudarthrose ein Polymethylmethacrylat-Spacer (PMMA-Spacer) eingebracht wird. Dieser Spacer ist meist mit Antibiotika behaftet und greift die Infektion so unmittelbar lokal an. Um diesen Spacer bildet sich ein Pseudoperiostschlauch, der dann in einer Folgeoperation mit Spongiosa befüllt werden kann [44]. Zu dieser Technik liegen noch keine groß angelegten vergleichenden Studien vor, jedoch konnten in einzelnen Fallstudien gute Ergebnisse erzielt werden [45,46]. Zusammengefasst ist diese Technik bei großem Defektareal und Osteomyelitis eine gute Option.

\section{Segmenttransport}

Der Segmenttransport kann mithilfe eines unilateralen Fixateurs oder eines Ringfixateurs durchgeführt werden. Ziel ist es, ein zuvor i. d. R. infiziertes Knochenareal zu entfernen und per Segmenttransport kontinuierlich wieder zuwachsen zu lassen. Indikationen für diese Technik sind daher Osteomyelitis und Infektpseudarthrosen. Bezüglich dieser Technik existieren einzelne Fallstudien am Unterarm, die gute Ergebnisse nach 6-7 Monaten aufweisen $[47,48]$. Dieses Verfahren erfordert eine gute Compliance seitens des Patienten, um ein Abrutschen des Fixateurs, eine Pin-Infektion und somit ein Therapieversagen zu vermeiden. Zusammengefasst kann diese Technik bei entsprechender Expertise des Operateurs, geeignetem Patienten und großem Defektareal als Option dienen.

\section{Revisionsstrategien bei fehlgeschla- genen Osteosynthesen - spezielle Verletzungen/Komplikationen}

\section{Galeazzi-Fraktur}

Indikationen für eine Revisionsoperation nach einer Galeazzi-Fraktur sind insgesamt selten. Eine Herausforderung in der Behandlung der Verletzung kann das distale Radioulnargelenk (DRUG) darstellen. Kommt es hier zu chronischen Instabilitäten, kann die Griffkraft und die Pronations- sowie Supinationsleistung eingeschränkt sein [49]. Eine Revision sollte dann erfolgen. Adressiert werden muss der trianguläre fibrokartilaginäre Komplex (TFCC) und die weiteren Stabilisatoren des DRUG. Der TFCC sollte weitestgehend rekonstruiert und das DRUG befundabhängig temporär fixiert werden [50]. Hierbei reicht es meist aus, das Gelenk mittels eines KirschnerDrahtes für 4-6 Wochen in Neutralstellung zu fixieren [51]. Eine intensive Nachbehandlungsphase mit physiotherapeutischer Beübung sollte sich anschließen.

Lässt sich das Gelenk nicht reponieren, ist meist von der Interposition von Weichteilen, wie der Sehne des M. extensor carpi ulnaris oder des M. extensor digiti minimi 

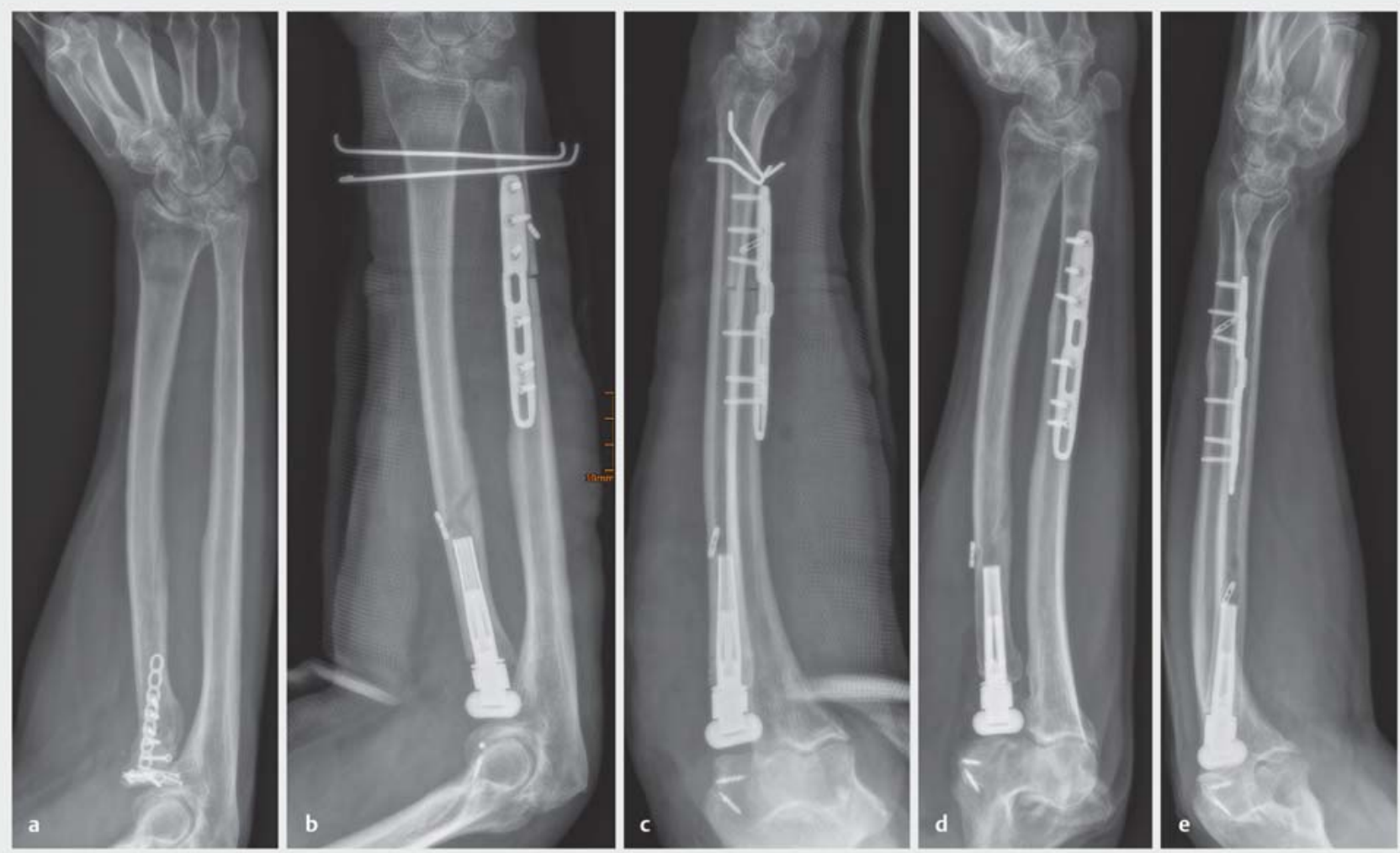

- Abb. 2 Bild einer Essex-Lopresti-Verletzung mit primärer fehlgeschlagener Plattenosteosynthese des proximalen Radius. a Deutliche Ulna-plusVariante mit abgerutschtem osteosynthetisch versorgtem Radiuskopf. b, c Unmittelbares postoperatives Ergebnis nach ME proximaler Radius, Radiuskopfprothese, Rekonstruktion des LUCL (lateral ulnar collateral ligament), Ulnaverkürzungsosteotomie mittels 2,5-mm-Verkürzungsplatte sowie LARS-Augmentation mit 2 Endobuttons (LARS: Ligament Augmentation and Repair System ${ }^{\circledR}$, Fa. Corin). d, e Ergebnis 24 Monate nach Revisionsoperation in a.-p. und lateraler Ansicht.

auszugehen. Eine offene Reposition muss in diesen Fällen erfolgen [50].

\section{Essex-Lopresti-Verletzung}

Eine Essex-Lopresti-Verletzung wird häufig im primären Verletzungsbild nicht erkannt und führt zu chronischer Instabilität des Unterarmes und Handgelenkbeschwerden durch eine Proximalisierung des Radius mit relativem Ulnavorschub und Radialdeviation der Hand ( $\mathbf{A} \mathbf{A b} \mathbf{b} . \mathbf{2} \mathbf{a}$ ) [6]. Da häufig im Falle einer primären Osteosynthese allenfalls eine Versorgung des Radiuskopfes vorliegt, ist die Revisionschirurgie meist von einer aufwendigen Rekonstruktion der Stabilisatoren des Unterarmes geprägt. Hierzu bedarf es einer Radiuskopfrevision, meist mittels Radiuskopfprothesenversorgung, einer Stabilisierung des distalen Radioulnargelenks mittels temporärer KirschnerDraht-Fixierung ( $\boldsymbol{A} \mathbf{A b b} . \mathbf{2} \mathbf{b}-\mathbf{c}$ ), offener Rekonstruktion des TFCC und zusätzlich häufig einer Ulnaverkürzungsosteotomie. Zusätzlich kann eine Augmentation des zentralen Bandes der Membrana interossea eine erneute Proximalisierung des Radius verhindern. Im eigenen Vorgehen erfolgt diese Bandrekonstruktion mittels eines sog. LARS-Bandes (LARS: Ligament Augmentation and Repair
System ${ }^{\circledR}$, Fa. Corin; > Abb. 2 b-e, 3 e, f) [52]. Dieses synthetische Band aus Polyethylen ist dabei als Augmentation der Membrana interossea zu verstehen. Zahlreiche Studien haben den anatomischen Verlauf dieser ligamentären Verbindung zwischen Ulna und Radius untersucht. Als besonders wichtig für die Stabilität haben sich hierbei die Fasern des zentralen Bandes herausgestellt [53]. Im Verlauf dieser Fasern wird daher das LARS-Band optimalerweise implantiert. Die Insertionspunkte und der Verlauf sind in > Tab. 1 zusammengefasst.

Radius und Ulna werden entsprechend dem physiologischen Verlauf und den Insertionspunkten des zentralen Bandes mit Bohrlöchern versehen und die Bandplastik über eine Knochenbrücke oder einen „Endobutton“ eng an den Knochen befestigt ( $\bullet$ Abb. $\mathbf{3}$ e, f). Um das Anhaften des Endobuttons am Knochen zu optimieren, kann kurzfristig die maximale Supination eingenommen werden. Als Alternativen zum LARS-Band können ein Patellar-Sehnen-Knochen-Implantat, ein Achillessehneninterponat sowie eine Palmaris-longus- oder Flexor-carpiradialis-Sehne als Autograft dienen [53,54]. Nachteil der 

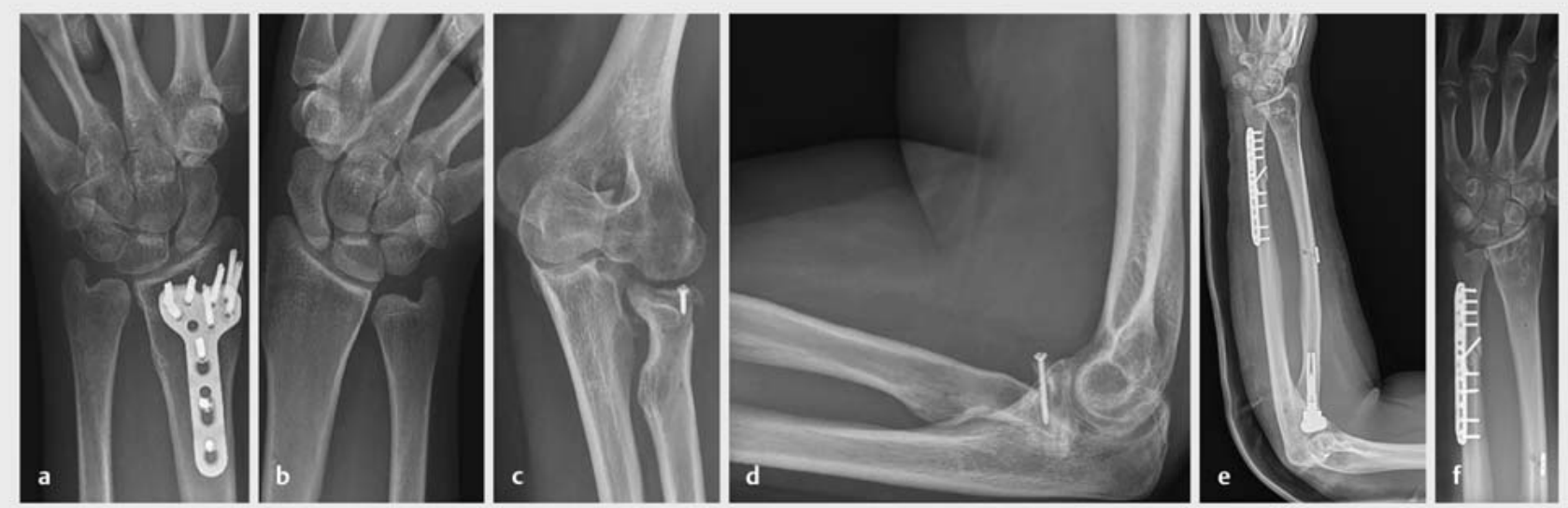

- Abb. 3 Erneutes Bild einer Essex-Lopresti-Verletzung mit primärer distaler und proximaler Radiusosteosynthese und Revisionsergebnis. a Diskrete Ulna-plus-Variante mit klinischen Beschwerden im Vergleich zur Gegenseite (b) im a.-p. Strahlengang. c, d Bild einer Radiuskopfnekrose mit extern bereits teilweise erfolgter ME, a.-p. und lateral. e, $\mathbf{f}$ Unmittelbares postoperatives Ergebnis nach ME distaler Radius, Ulnaverkürzungsosteotomie mittels 2,5-mm-Verkürzungsplatte, LARS-Augmentation mit 2 Endobuttons und Radiuskopfprothese sowie Rekonstruktion des LUCL; Korrektur der Ulna-plus-Variante in (f) sichtbar.

- Tab. 1 Insertionspunkte und Verlauf des Zentralbandes der Membrana interossea [53,54].

\begin{tabular}{|l|l|}
\hline Ulna & $\begin{array}{l}\text { Schnittstelle zwischen distalem und } \\
\text { mittlerem Drittel; ca. } 6 \mathrm{~cm} \text { proximal des } \\
\text { Processus styloideus ulnae (PSU) }\end{array}$ \\
\hline Radius & $\begin{array}{l}\text { Punkt } 60 \% \text { der Länge des Radius vom } \\
\text { Processus styloideus radii (PSR) gemes- } \\
\text { sen; } \text { ca. } 12 \mathrm{~cm} \text { proximal des PSR }\end{array}$ \\
\hline $\begin{array}{l}\text { Orientierung } \\
\text { der Fasern }\end{array}$ & $\begin{array}{l}\text { vom Radius aus: } 21^{\circ} \text { in Richtung langer } \\
\text { Schaftachse der Ulna }\end{array}$ \\
\hline
\end{tabular}

Autografts kann eine geringere Steifigkeit unter Belastung sein [53].

Die Ulnaverkürzungsosteotomie sollte schließlich mittels einer Kompressionsplatte 2,5-3,5 mm gehalten werden.

Als „Salvage Procedures“ sind die Sauvé-Kapandji-Methode und die operative Schaffung einer radioulnaren Synostose zu nennen. Bei der Sauvé-Kapandji-Methode wird eine Arthrodese des DRUG sowie eine Segmentresektion der distalen Ulna durchgeführt. Hierdurch wird versucht, dem Patienten die Umwendbewegung des Unterarmes durch Proximalisierung des Drehzentrums nach proximal-ulnar wieder zu ermöglichen. Diese Methoden stellen die Funktion nicht ausreichend gut wieder her und sollten daher nur bei multiplen Voroperationen, ausgeprägtem Leidensdruck und Dysfunktion Anwendung finden. Die postoperativen Ergebnisse dieser Verfahren sind in der Literatur nur schwer zu vergleichen, da die Studienpopulationen sehr heterogen sind mit einer großen Bandbreite an verschiedenen Indikationen [53].

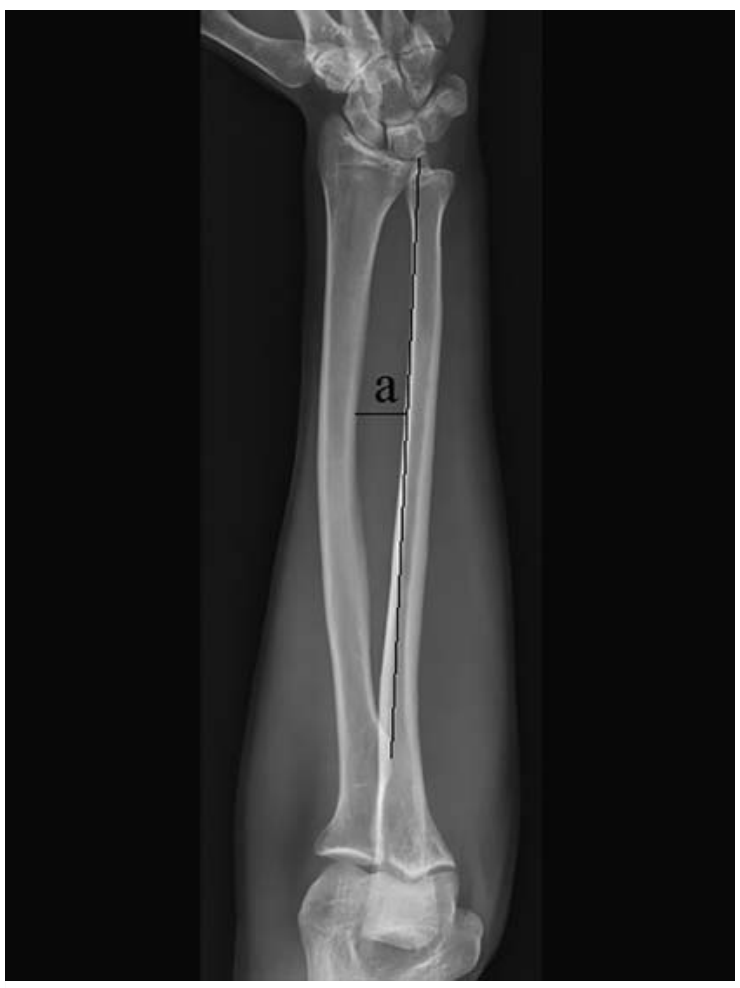

- Abb. 4 Darstellung des physiologischen radialen Bogens, an dem sich die Rekonstruktion des Unterarmes orientieren sollte. Es wird eine Linie von der Tuberositas radii bis zum distalen Radioulnargelenk (DRUG) gezogen; „a“ markiert die maximale Höhe des radialen Bogens. 


\section{Malunion}

Die Anatomie des Unterarmes muss möglichst genau rekonstruiert werden, da kleinste Abweichungen erhebliche Auswirkungen auf die Funktion haben können. Im Rahmen einer fehlverheilten Unterarmfraktur mit klinisch manifestem Funktionsverlust ist es dienlich, den Unterarm der Gegenseite als anatomische Schablone zu nutzen, da es keine festen Normvarianten gibt [14]. Im Detail ist hierbei auf den radialen Bogen zu achten und dieser möglichst genau zu rekonstruieren ( $>$ Abb. 4 ). Die zu planende Osteotomie ist als 3-dimensionale Operation, unter Nutzung der kommerziell erhältlichen patientenspezifischen Instrumentarien (Schablonen), durchzuführen. Die Ergebnisse sind zufriedenstellend, wobei der Zeitpunkt der Korrekturosteotomie entscheidend ist. Patienten, die innerhalb von 12 Monaten nach initialer Fraktur operiert wurden, haben eine signifikant bessere Funktion, als Patienten, die später korrigiert wurden [55].

\section{Kernaussagen}

In der Revisionschirurgie des Unterarmes ist die genaue anatomische und funktionelle Kenntnis der knöchernen und ligamentären Strukturen Grundvoraussetzung für eine erfolgreiche Operation. Pseudarthrosen bilden den Großteil der Indikationen für eine Revisionsoperation am Unterarm. Hierbei empfiehlt sich ein lokales Débridement, kombiniert mit einer Augmentation und stabilen Plattenosteosynthese. Bei allen weiteren möglichen Komplikationen einer primären Osteosynthese (Malunion, spezielle Frakturformen) ist ein individuell angepasstes Vorgehen notwendig.

\section{Interessenkonflikt}

Andreas Harbrecht: kein Interessenskonflikt Michael Hackl: Referententätigkeit für Arthrex; Drittmittelprojekt mit der IBRA (International Bone Research Association) Kilian Wegmann: Bezahlter Berater für Medartis, Arthrex; Finanzielle wissenschaftliche Unterstützung durch Medartis, Arthrex, Tornier, Stryker

Lars Peter Müller: Bezahlter Berater für Medartis, Arthrex, Tornier; Finanzielle wissenschaftliche Unterstützung durch Medartis, Arthrex, Tornier, Stryker

\section{Autorinnen/Autoren}

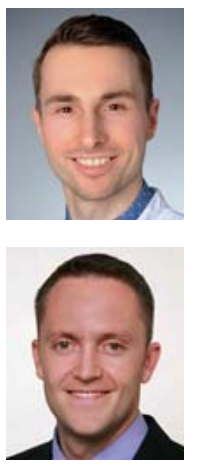

\section{Andreas Harbrecht}

Dr. med., Assistenzarzt, Klinik und Poliklinik für Orthopädie und Unfallchirurgie, Schwerpunkt für Unfall-, Hand-, und Ellenbogenchirurgie, Uniklinik Köln

\section{Michael Hackl}

Dr. med., Funktionsoberarzt, Klinik und Poliklinik für Orthopädie und Unfallchirurgie, Schwerpunkt für Unfall-, Hand-, und Ellenbogenchirurgie, Uniklinik Köln

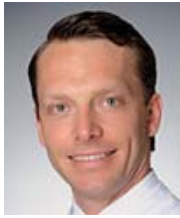

\section{Kilian Wegmann}

PD Dr. med., Leitender Oberarzt, Klinik und Poliklinik für Orthopädie und Unfallchirurgie, Schwerpunkt für Unfall-, Hand-, und Ellenbogenchirurgie, Uniklinik Köln

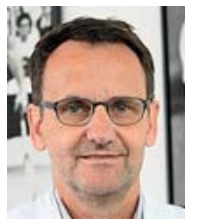

Lars Peter Müller

Univ.-Prof. Dr. med., Stellvertretender Direktor, Klinik und Poliklinik für Orthopädie und Unfallchirurgie, Schwerpunkt für Unfall-, Hand-, und Ellenbogenchirurgie, Uniklinik Köln

\section{Korrespondenzadresse}

Dr. med. Andreas Harbrecht

Klinik und Poliklinik für Orthopädie und Unfallchirurgie, Schwerpunkt für Unfall-, Hand-, und Ellenbogenchirurgie Uniklinik Köln

Kerpener Straße 62

50937 Köln

Tel.: $0221 / 478-0$

Fax: 0221/478-4835

andreas.harbrecht@uk-koeln.de

\section{Literatur}

[1] Birkbeck DP, Failla JM, Hoshaw SJ et al. The interosseous membrane affects load distribution in the forearm. J Hand Surg Am 1997; 22: 975-980. doi:10.1016/S0363-5023(97)80035-4

[2] Kusswetter W, Schmid K. [Membrana interossea antebrachii a common ligament of the radius-ulna joint. II: submacroscopic and microscopic structure]. Z Orthop Ihre Grenzgeb 1979; 117: 776-783

[3] McGinley JC, Kozin SH. Interosseous membrane anatomy and functional mechanics. Clin Orthop Relat Res 2001; (383): 108 122. doi:10.1097/00003086-200102000-00013

[4] Ellwein A, Lill H. [Diaphyseal fractures of the forearm in adults]. Obere Extremität 2015; 10: 222-228. doi:10.1007/ s11678-015-0338-9

[5] Josten C, Marquaß B. Frakturen, Luxationen, Bandrupturen Ellenbogen, Unterarm. Orthopädie und Unfallchirurgie up2date 2006; 1: 447-476. doi:10.1055/s-2006-944805

[6] Essex-Lopresti P. Fractures of the radial head with distal radioulnar dislocation; report of two cases. J Bone Joint Surg $\mathrm{Br}$ 1951; 33 B: 244-247

[7] Court-Brown CM, Caesar B. Epidemiology of adult fractures: a review. Injury 2006; 37: 691-697. doi:10.1016/j.injury.2006. 04.130

[8] Marcheix PS, Delclaux S, Ehlinger M et al. Pre- and postoperative complications of adult forearm fractures treated with plate fixation. Orthop Traumatol Surg Res 2016; 102: 781784. doi:10.1016/j.otsr.2016.04.014

[9] Chung KC, Spilson SV. The frequency and epidemiology of hand and forearm fractures in the United States. J Hand Surg Am 2001; 26: 908-915. doi:10.1053/jhsu.2001.26322

[10] Jones DB jr., Kakar S. Adult diaphyseal forearm fractures: intramedullary nail versus plate fixation. J Hand Surg Am 2011; 36 : 1216-1219. doi:10.1016/j.jhsa.2011.03.020

[11] Chapman MW, Gordon JE, Zissimos AG. Compression-plate fixation of acute fractures of the diaphyses of the radius and 
ulna. J Bone Joint Surg Am 1989; 71: 159-169. doi:10.2106 00004623-198971020-00001

[12] Wright RR, Schmeling G], Schwab JP. The necessity of acute bone grafting in diaphyseal forearm fractures: a retrospective review. J Orthop Trauma 1997; 11: 288-294. doi:10.1097| 00005131-199705000-00012

[13] Wei SY, Born CT, Abene A et al. Diaphyseal forearm fractures treated with and without bone graft. J Trauma 1999; 46: 1045-1048. doi:10.1097/00005373-199906000-00011

[14] Schemitsch EH, Richards RR. The effect of malunion on functional outcome after plate fixation of fractures of both bones of the forearm in adults. J Bone Joint Surg Am 1992; 74: 10681078. doi:10.2106/00004623-199274070-00014

[15] Hadden WA, Reschauer R, Seggl W. Results of AO plate fixation of forearm shaft fractures in adults. Injury 1983; 15: 4452

[16] Ross ER, Gourevitch D, Hastings GW et al. Retrospective analysis of plate fixation of diaphyseal fractures of the forearm bones. Injury 1989; 20: 211-214

[17] Anderson LD, Sisk D, Tooms RE et al. Compression-plate fixation in acute diaphyseal fractures of the radius and ulna. J Bone Joint Surg Am 1975; 57: 287-297

[18] Ring D, Allende C, Jafarnia K et al. Ununited diaphyseal forearm fractures with segmental defects: plate fixation and autogenous cancellous bone-grafting. J Bone Joint Surg Am 2004; 86: 2440-2445

[19] Stern PJ, Drury W]. Complications of plate fixation of forearm fractures. Clin Orthop Relat Res 1983; (1983): 25-29

[20] Price CT, Knapp DR. Osteotomy for malunited forearm shaft fractures in children. J Pediatr Orthop 2006; 26: 193-196. doi:10.1097/01.bpo.0000194699.29269.76

[21] Zura R, Mehta S, Della Rocca G] et al. Biological risk factors for nonunion of bone fracture. JBJS Rev 2016; 4. doi:10.2106/JBJS. RVW.0.00008

[22] Hernandez RK, Do TP, Critchlow CW et al. Patient-related risk factors for fracture-healing complications in the United Kingdom General Practice Research Database. Acta Orthop 2012; 83: 653-660. doi:10.3109/17453674.2012.747054

[23] Ricci WM, Streubel PN, Morshed S et al. Risk factors for failure of locked plate fixation of distal femur fractures: an analysis of 335 cases. J Orthop Trauma 2014; 28: 83-89. doi:10.1097| BOT.0b013e31829e6dd0

[24] Patel RA, Wilson RF, Patel PA et al. The effect of smoking on bone healing: A systematic review. Bone Joint Res 2013; 2: 102-111. doi:10.1302/2046-3758.26.2000142

[25] dos Reis FB, Faloppa F, Fernandes H] et al. Outcome of diaphyseal forearm fracture-nonunions treated by autologous bone grafting and compression plating. Ann Surg Innov Res 2009; 3: 5. doi:10.1186/1750-1164-3-5

[26] Brakenbury PH, Corea JR, Blakemore ME. Non-union of the isolated fracture of the ulnar shaft in adults. Injury 1981; 12: 371-375

[27] Ring D, Rhim R, Carpenter C et al. Comminuted diaphyseal fractures of the radius and ulna: does bone grafting affect nonunion rate? J Trauma 2005; 59: 436-440. doi:10.1097/01. ta.0000174839.23348.43

[28] Stevens CT, ten Duis HJ. Plate osteosynthesis of simple forearm fractures: LCP versus DC plates. Acta Orthop Belg 2008; 74: $180-183$

[29] Hofmann A, Hessmann MH, Rudig L et al. [Intramedullary osteosynthesis of the ulna in revision surgery]. Unfallchirurg 2004; 107: 583-592. doi:10.1007/s00113-004-0790-2
[30] Kloen P, Wiggers JK, Buijze GA. Treatment of diaphyseal nonunions of the ulna and radius. Arch Orthop Trauma Surg 2010; 130: 1439-1445. doi:10.1007/s00402-010-1071-x

[31] Faldini C, Pagkrati S, Nanni M et al. Aseptic forearm nonunions treated by plate and opposite fibular autograft strut. Clin Orthop Relat Res 2009; 467: 2125-2134. doi:10.1007/s11999009-0827-5

[32] Spira E. Bridging of bone defects in the forearm with iliac graft combined with intramedullary nailing. J Bone Joint Surg $\mathrm{Br}$ 1954; 36-B: 642-646

[33] Davey PA, Simonis RB. Modification of the Nicoll bone-grafting technique for nonunion of the radius and/or ulna. J Bone Joint Surg $\operatorname{Br} 2002 ; 84$ : 30-33

[34] Moroni A, Rollo G, Guzzardella M et al. Surgical treatment of isolated forearm non-union with segmental bone loss. Injury 1997; 28: 497-504

[35] Cox G, Jones E, McGonagle D et al. Reamer-irrigator-aspirator indications and clinical results: a systematic review. Int Orthop 2011; 35: 951-956. doi:10.1007/s00264-010-1189-z

[36] Allsopp BJ, Hunter-Smith DJ, Rozen WM. Vascularized versus nonvascularized bone grafts: what is the evidence? Clin Orthop Relat Res 2016; 474: 1319-1327. doi:10.1007/s11999016-4769-4

[37] Wood MB. Upper extremity reconstruction by vascularized bone transfers: results and complications. J Hand Surg Am 1987; 12: 422-427

[38] Safoury Y. Free vascularized fibula for the treatment of traumatic bone defects and nonunion of the forearm bones. J Hand Surg $\mathrm{Br} 2005$; 30: 67-72. doi:10.1016/j.jhsb.2004. 09.007

[39] Kamrani RS, Farhoud A, Nabian MH et al. Vascularized posterior interosseous pedicled bone grafting for infected forearm nonunion. J Hand Surg Eur Vol 2016; 41: 441-447. doi: $10.1177 / 1753193415601041$

[40] Vicenti G, Maruccia M, Carrozzo M et al. Free vascularized osteoseptocutaneous fibular flap for radius shaft nonunion: The final solution when the iliac crest autograft fails. A case report. Injury 2018; 49 (Suppl. 4): S63-S70. doi:10.1016/j.injury. 2018.11.030

[41] Dell PC, Sheppard JE. Vascularized bone grafts in the treatment of infected forearm nonunions. J Hand Surg Am 1984; 9: 653-658

[42] Jupiter JB, Gerhard HJ, Guerrero J et al. Treatment of segmental defects of the radius with use of the vascularized osteoseptocutaneous fibular autogenous graft. J Bone Joint Surg Am 1997; 79: 542-550. doi:10.2106/00004623-19970400000009

[43] Finkemeier CG. Bone-grafting and bone-graft substitutes. J Bone Joint Surg Am 2002; 84: 454-464. doi:10.2106/ 00004623-200203000-00020

[44] Masquelet AC, Begue T. The concept of induced membrane for reconstruction of long bone defects. Orthop Clin North Am 2010; 41: 27-37; table of contents. doi:10.1016/j.ocl.2009. 07.011

[45] Micev AJ, Kalainov DM, Soneru AP. Masquelet technique for treatment of segmental bone loss in the upper extremity. J Hand Surg Am 2015; 40: 593-598. doi:10.1016/j.jhsa.2014. 12.007

[46] Walker M, Sharareh B, Mitchell SA. Masquelet reconstruction for posttraumatic segmental bone defects in the forearm. J Hand Surg Am 2019; 44: 342.e1-342.e8. doi:10.1016/j. jhsa.2018.07.003 
[47] El-Mowafi H, Elalfi B, Wasfi K. Functional outcome following treatment of segmental skeletal defects of the forearm bones by Ilizarov application. Acta Orthop Belg 2005; 71: 157-162

[48] Zhang Q, Yin P, Hao M et al. Bone transport for the treatment of infected forearm nonunion. Injury 2014; 45: 1880-1884. doi:10.1016/j.injury.2014.07.029

[49] Strehle J, Gerber C. Distal radioulnar joint function after Galeazzi fracture-dislocations treated by open reduction and internal plate fixation. Clin Orthop Relat Res 1993; (293): 240245

[50] Rothe M, Rudy T, Stanković P et al. [Treatment of Galeazzi's fracture - is the surgical revision of the distal radioulnar joint necessary?]. Handchir Mikrochir Plast Chir 2001; 33: 252-257. doi:10.1055/s-2001-16587

[51] Morgan W], Breen TF. Complex fractures of the forearm. Hand Clin 1994; 10: 375-390

[52] Sabo MT, Watts AC. Reconstructing the interosseous membrane: a technique using synthetic graft and endobuttons.
Tech Hand Up Extrem Surg 2012; 16: 187-193. doi:10.1097| BTH.0b013e3182634ce4

[53] Phadnis ], Watts AC. Longitudinal instability of the forearm. Orthopade 2016; 45: 861-869. doi:10.1007/s00132-0163329-7

[54] Adams JE, Culp RW, Osterman AL. Central band interosseous membrane reconstruction for forearm longitudinal instability. J Wrist Surg 2016; 5: 184-187. doi:10.1055/s-0036-1585070

[55] Trousdale RT, Linscheid RL. Operative treatment of malunited fractures of the forearm. J Bone Joint Surg Am 1995; 77: 894902. doi:10.2106/00004623-199506000-00010

\section{Bibliografie}

DOI https://doi.org/10.1055/a-1004-3735

online publiziert 03.12.2019 | OP-JOURNAL 2020; 36: 124132 @ Georg Thieme Verlag KG Stuttgart · New York ISSN 0178-1715 